\title{
TENDENCIAS Y APLICACIONES DE LAS TIC EN LA EDUCACIÓN PERMANENTE DE PERSONAS MAYORES
}

\author{
Ana Manzano-León \\ manzleon@gmail.com \\ David Padilla-Góngora \\ José Manuel Aguilar-Parra \\ Pilar Díaz-López \\ Universidad de Almería
}

Fecha de Recepción: 22 Junio 2018

Fecha de Admisión: 1 Octubre 2018

\section{RESUMEN}

En esta investigación pretendemos mostrar las últimas tendencias de las tecnologías de la información y la comunicación (TIC) y su aplicación en la educación permanente de personas mayores, del mismo modo estudiamos cómo el uso de las TIC tiene una serie de beneficios académicos, psicológicos y sociales en las personas mayores, en definitiva, como mejoran su calidad de vida.

Palabras clave: competencias digitales; formación TIC; envejecimiento activo

\section{ABSTRACT}

TRENDS AND APPLICATIONS OF ICT IN THE PERMANENT EDUCATION OF OLDER PEOPLE

In this research, we pretend to investigate the latest trends in Information and Communications Technology (ICT) and its application in the continuing education of eldery people, the same way we study how the use of ICT has a number of academic, psychological and social benefits in the elderly, and ultimately improve their quality of life.

Keywords: digital competences; ICT training; active aging

\section{ANTECEDENTES DE LAS TIC EN LA EDUCACIÓN PERMANENTE DE PERSONAS MAYORES}

La combinación de las TIC (tecnologías de la información y comunicación) y las personas mayores se describe a menudo en términos problemáticos. La vejez a menudo implica una "desconexión" de diferentes áreas sociales, como el campo de la tecnología y la participación política y ciudadana. La brecha digital, obviamente, tiene una división relacionada con la edad, siendo las personas mayores las más representadas dentro de esa brecha, debido a dos factores principales: En primer lugar por factores biológicos, cognitivos, psicológicos, sociales y económicos que pueden formar una barrera para la adopción de las TIC (como por ejemplo, pérdida de visión, una capacidad de aprender y retener información sobre nuevos temas en declive, la reducción contacto con 
ambientes que ofrecen las nuevas tecnologías (por ejemplo, el lugar de trabajo), y limitaciones financieras); y en segundo lugar, los antecedentes sociohistóricos de las personas mayores que crecieron en una sociedad que estaba dominada por los medios de comunicación en un solo sentido (imprenta y radio), y por lo tanto carecen de recursos y hábitos necesarios para tratar adecuadamente las nuevas TIC (Steyaert, Eggermont y Vandebosch, 2006). Sin embargo, las TIC se están convirtiendo en una excelente herramienta para contribuir al desarrollo de un envejecimiento activo adecuado.

Las competencias digitales de las personas mayores están en constante aumento, pero no al mismo ritmo que las generaciones más jóvenes (Esteller y Escuder, 2012), ya que como afirma Prensky (2010) constituyen las primeras generaciones formadas en los nuevos avances tecnológicos, con los que tienen un sentimiento de pertenencia y "natividad digital"; En cambio, las personas mayores utilizan sólo los aspectos más básicos de las TIC. Benito (2009) considera este factor muy relevante a la hora de realizar programas socioeducativos de educación permanente en personas mayores, ya que el docente debe tener en cuenta que las personas más jóvenes suelen ser grupos más homogéneos y van más rápidas en aspectos como: movimientos, reacciones ante la tecnología, autonomía y velocidad de uso, etc. Sin embargo, en los grupos de personas mayores hay grandes diferencias según su cultura, personalidad, historia de vida, escolarización, habilidades digitales y capacidades físicas y cognitivas.

Las TIC pueden ser un elemento determinante del envejecimiento activo de una persona mayor. El significado de "activo/a" está directamente relacionado con el uso que les dé a herramientas TIC como el ordenador, el teléfono móvil, tablets, cámara, Internet, etc., no sólo a su uso instrumental, también refleja la capacidad de ser consciente de la realidad y su capacidad de actuar e influir en la red (Esteller, Escuder y Traver, 2009 citado por Manzano, Aguilar y López, 2016)

Morales (2013) profundiza en la necesidad de una educación permanente actualizada, que cuente con las herramientas TIC necesarias para que las personas mayores puedan adquirir las competencias digitales necesarias para poder seguir siendo actualmente ciudadanos y ciudadanas autónomas, consiguiendo así un empoderamiento para acceder fácilmente a la información en la red, realizar tareas cotidianas como tener actualizadas de manera online las cuentas bancarias, pedir citas previas en administraciones públicas y privadas... en definitiva, seguir siendo partícipes en la sociedad moderna, y consecuentemente mejorar su calidad de vida.

De Hert y Gutwirth (2006) señalan algunas herramientas de tecnología de asistencia que ya se están utilizando por y para las personas mayores como son: computación afectiva, asistencia de la memoria, robótica, inteligencia artificial, sensores, formación TIC para el desarrollo físico y cognitivo, interacción cerebro-ordenador, sistemas de navegación, informática sanitaria, interacción con dispositivos (usuarios virtual y artefactos virtuales), redes sociales, traductores de idiomas, comunidades virtuales de aprendizaje y comunidades y videojuegos online.

Las tecnologías de la información y la comunicación permiten la recolección, sistematización y difusión de información con el fin de promover el autoconocimiento, el aprendizaje y habilidades en las personas mayores (Struk y Moss, 2009), lo que disminuye las barreras de accesibilidad y mejora su calidad de vida (Weiner, Cudney y Winters, 2005). Chaparro y Barrera (2013) muestran que, a pesar de existir esta tecnología y sus posibilidades para mejorar la calidad de vida de las personas mayores, aún no se han utilizado de manera suficientemente sistemática, ni se realiza suficiente formación para la alfabetización digital no sólo instrumental, si no para la participación a través de las TIC. 


\section{OBJETIVOS DE LA INVESTIGACIÓN}

Realizar una revisión de aplicaciones innovadores de las TIC dentro del ámbito de la educación permanente.

Identificar los beneficios del uso de las TIC y las redes sociales en personas mayores.

\section{METODOLOGÍA}

Se ha realizado una revisión sistemática de artículos científicos consultando las bases de datos Web of Knowledge, Researchgate, Dialnet y Scopus, con restricción de fecha (2010/2016), en los idiomas español e inglés. No se hicieron restricciones respecto al tipo de estudio. Se revisaron los resúmenes y en los casos necesarios los artículos completos, teniéndose en cuenta finalmente todos los artículos que incluían programas de enseñanza-aprendizaje en línea y aplicaciones de las TIC innovadoras que mostrasen beneficios en la formación permanente de las personas mayores, eliminando el resto.

\section{RESULTADOS ALCANZADOS}

Tras la revisión bibliográfica, nos centramos principalmente en cinco nuevas tendencias de uso de las TIC en educación permanente:

\section{Tabla 1}

Tendencias de uso de TIC en educación permanente para personas mayores

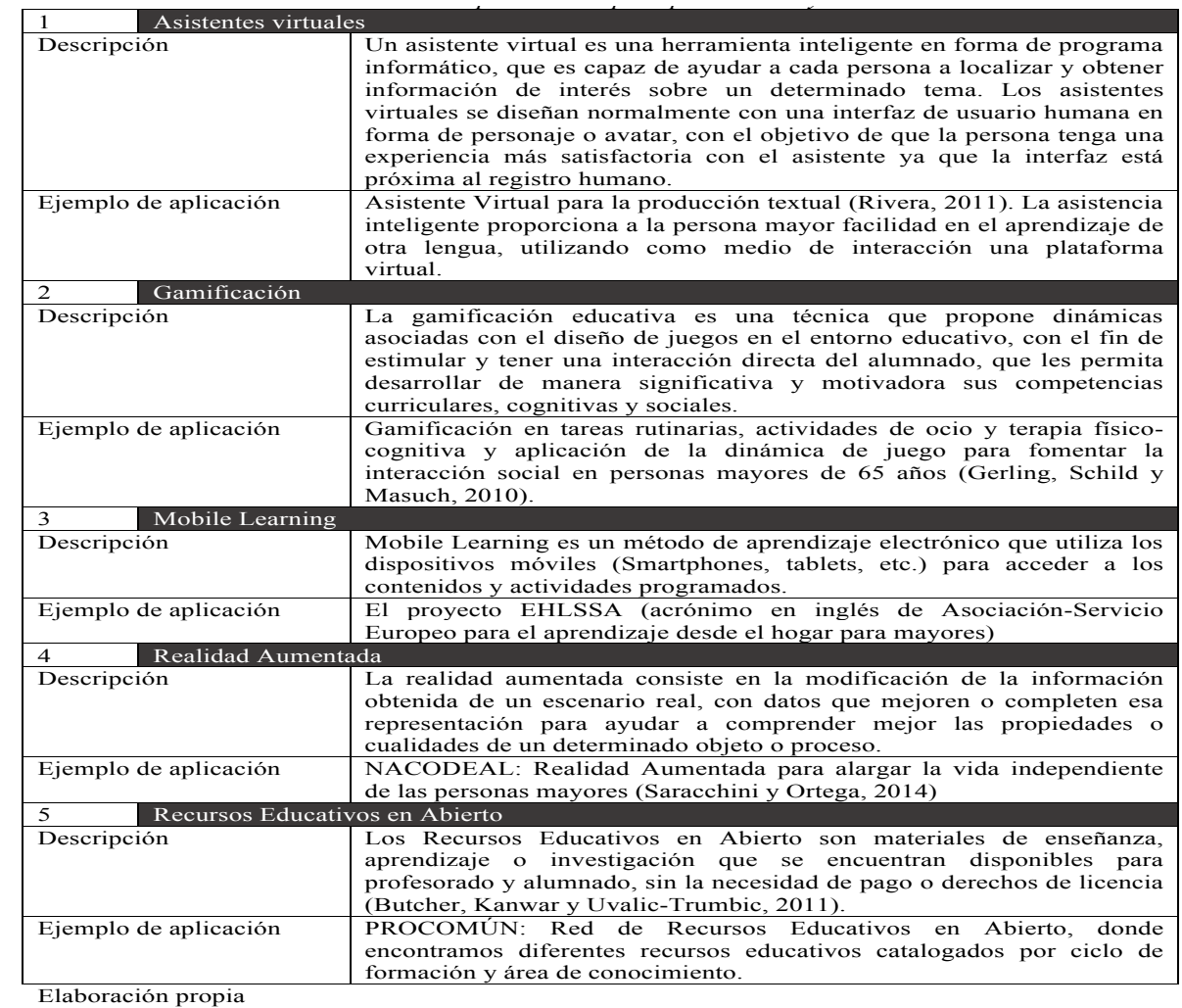




\section{TENDENCIAS Y APLICACIONES DE LAS TIC EN LA EDUCACIÓN PERMANENTE DE PERSONAS MAYORES}

\section{CONCLUSIONES}

Las tecnologías de la información y la comunicación (TIC) contribuyen a la mejora de la difusión de conocimiento, las relaciones sociales (amistades, familiares, etc.), los programas de educación permanente de las personas mayores tanto presenciales como e-learning, y la innovación en los servicios públicos destinados a las personas mayores.

Las TIC no son estáticas, es un fenómeno en continuo cambio y crecimiento emergente, siendo su trasfondo el crecimiento de la tecnología, desde ordenadores a teléfonos inteligentes, tabletas, y su uso no intrusivo en educación. Estas herramientas permiten no sólo la investigación sino también puede proporcionar las condiciones para que el conocimiento sea compartido y socializado, convirtiendo así en una herramienta clave para trabajar el envejecimiento activo.

El uso de las tecnologías de la información y la comunicación ha llegado a desarrollar propuestas pedagógicas que contribuyen a la construcción del conocimiento, facilitando así nuevos contextos de interacción entre personas y producen un cambio en la enseñanza-aprendizaje, Lin, Tsai, Chai y Lee (2013) definen el aprendizaje actual como el proceso de construcción de redes de información, contacto y recursos que se aplican a los problemas reales, que puede estar distribuido mediante las herramientas tecnológicas, en los denominados nodos de información.

Sin embargo, para poder utilizar estas herramientas tecnológicas, es necesario realizar programas de alfabetización digital y competencias digitales, reduciendo así la brecha digital en el colectivo de personas mayores. La brecha digital no se caracteriza únicamente como una consecuencia de las variables socioeconómicas, también influyen las motivaciones personales, la dinámica social y los elementos culturales (Demunter, 2005).

Reducir la brecha digital significaría fortalecer los hilos del tejido social y que la sociedad adquiriera una serie de beneficios como un mejor desarrollo económico, mejoras en la atención médica y unos niveles de inclusión social mejorados. Desde el punto de vista tecnológico, estos cambios pueden fomentar la creación de aplicaciones altamente accesibles, que permitan involucrar y motivar a las personas a interactuar con las TIC y mejorar su vida personal y sus roles dentro de la familia y la comunidad (Manzano, Aguilar y López, 2016).

\section{REFERENCIAS BIBLIOGRÁFICAS}

Barrera L, Pinto N, Sánchez B (2007). Resarch network Network of Researchers on Caring for Chronic Patient Caregivers. Aquichan, 7(2), 199-206

Benito, D. (2009). Aprendizaje en el entorno del e-learning: estrategias y figura del e-moderador. RUSC. Revista de Universidad y Sociedad del Conocimiento, 6 (2), 3-9.

Butcher, N., Kanwar, A., y Uvalic-Trumbic, S. (2011). A basic guide to open educational resources (OER). Vancouver, Canada: Commonwealth of Learning, and Paris, France: UNESCO. Recuperado de: http://www.col.org/oerBasicGuide

Chadwick, A., Tedesco, D., Tullis, T. (2004). Older Adults and Web Usability: is Web Experience the Same as Web Expertise? Extended Abstracts of the 2004 Conference on Human Factors and Computing Systems, 1391-1394 Vienna, Austria.

Chaparro, L. y Barrera, L. (2013). ICT as a Social Support Mechanism for Family Caregivers of People with Chronic Illness: A Case Study. Aquichan, 13 (1), 27-40.

De Hert, P. y Gutwirth, S. (2006). Privacy, data protection and law enforcement: Opacity of the individual and transparency of power. In E. Claes, A. Duff, \& S. Gutwirth (Eds.), Privacy and the criminal law. Antwerpen, Oxford: Intersentia.

Demunter C. (2005). The digital divide in Europe. Report number: 38/2005, Statistical Office of the European Communities, Luxembourg.

Esteller, R. y Escuder, P. (2012). Non-practical ICT courses for seniors for a comprehensive invol- 
vement to provide a global understanding of the Knowledge

Society. Social and Behavioral Sciences, 46, $2356-2361$.

Gerling, K., Schild, J., y Masuch, M. (2010). Exergame Design for Elderly Users: The Case Study of SilverBalance. International Conference on Advances in Computer Entertainment Technology 2010, Taipei, Taiwan.

Lin, T., Tsai, C., Chai, C. y Lee, M. (2013). Identifying Science Teachers' Perceptions of Technological Pedagogical and Content Knowledge (TPACK). Journal of Science Education and Technology, 22 (3), 325-336.

Manzano, A., Aguilar, J., López, R. (2016). Las TIC como determinantes del envejecimiento activo. $7^{\text {th }}$ International Conference on Intercultural Education "Education, Health, and ICTs from a Transcultural Perspective".

Morales, P. (2013). El uso de las TIC y la formación permanente del adulto: una mejora de la calidad de vida. Ariadna; cultura, educación y tecnología, 1(1), 58-62. D0l: http://dx.doi.org/10.6035/Ariadna.2013.1.9

Prensky, M. (2010). Nativos e inmigrantes digitales. Distribuidora SEK, S.A. Impresión: Albatros, S.L. Recuperado de:

http://www.marcprensky.com/writing/Prensky-NATIVOS\%20E\%20INMIGRANTES\%20DIGITALES\%20(SEK).pdf

Rivera, D. (2011). Asistente Virtual Para La Producción Textual (AVPT) (Tesis doctoral). Universidad del Valle, Cali.

Saracchini, R. y Ortega, C. (2014). An Easy to Use Mobile Augmented Reality Platform for Assisted Living Using Pico-projectors. Computer Science, 8671, 552-561. DOI: http://dx.doi.org/10.1007/978-3-319-11331-9 66

Struk C. y Moss, J. (2009). Focus on Technology: What Can You Do to Move the Vision Forward? Computers Informatics Nursing, 27(3), 192-194.

Weiner C, Cudney S, Winters C. (2005) Social Support in Cyberspace. The next Generation. Computers Informatics Nursing, 23(1), 7- 15. 
